www.jmscr.igmpublication.org

Impact Factor 5.84

Index Copernicus Value: 83.27

ISSN (e)-2347-176x ISSN (p) 2455-0450

crossref DOI: https://dx.doi.org/10.18535/jmscr/v5i7.143

Journal Of Medical Science And Clinical Research

IGM Publication

An Official Publication of IGM Publication

\title{
Imaging of Adrenal Tuberculosis as Bilateral Enlargement
}

\author{
Authors \\ Dr Kirti Rana (Professor), Dr Om Prakash Rathore (Resident Doctor) \\ Dr R.N. Gehlot (Professor) \\ Department of Radiodiagnosis, Dr. S.N. Medical Collage Jodhpur (Raj)
}

\section{INTRODUCTION}

Adrenal tuberculosis is caused by Mycobacterium tuberculosis complex and spreads by hematogeneous route. Clinical manifestations may take years to become apparent, and asymptomatic infection is not uncommon. Adrenal involvement was found in $6 \%$ of patients with active tuberculosis. The majority of patients with active or recently acquired disease $(<2$ years $)$ have bilateral adrenal enlargement, while calcification and atrophy are the norm with more remote infection or inactive disease. The adrenals can be enlarged in patients with pulmonary tuberculosis without active involvement of the glands. Stress and inflammation could be potential reasons. Adrenal biopsy is not necessary for primary adrenal insufficiency with bilateral adrenal enlargement in a patient with proven extra-adrenal tuberculosis. The widespread use of computed tomography has improved our understanding of the patterns of involvement of the adrenal gland in tuberculosis.

\section{CASE REPORT}

A $50 Y$ old man presented to his physician 3month back with generalised weakness, fatigue and history of progressive black discolouration of skin, ear lobules, lips and palate and complained of loss of appetite, weight loss. Patient also consulted a private practitioner for recurrent episodes of hypotension. The cortisol levels were reduced and subsequently treated as Addison crisis by steroids by private practitioner. However, the hypotension is not controlled with steroids. Hence patient was referred to medicine department.

GPE: Patient had low blood pressure (110/60 despite steroid treatment), muscle weakness and hyperpigmented skin.

Blood investigation: CBC: Normal, RFT: mild Deranged (increased urea and creatinin), TFT: Normal, Electrolytes: Low sodium, ACTH: Normal, Cortisol: normal (patient on steroid).

Radiological investigations

1) X-Ray: Calcification at the anatomic location of adrenal gland on X-ray

Calcified lymph nodes.

Small left kidney with calcifications on X-Ray

2) Ultrasonography: Left kidney shows reduced blood flow on colour Doppler.

3) CT urography: Right Kidney \& Ureter: Normal in shape and size.

Left Kidney \& Ureter: Small and contracted with cortical scaring and medullary \& papillary 
calcification, mainly in mid and lower pole. No $\mathrm{E} / \mathrm{O}$ contrast excretion on delayed scan.

\section{4) CECT abdomen}

Adrenal gland shows bilateral symmetrical enlargement and associated with dot like calcifications.

Left Kidney is relatively small and shows no nephrographic density and absent contrast excretion in delayed scan.

Few calcified lymph nodes.

\section{5) Treatment and follow up}

During hospital stay also his hypotension could not be controlled only on steroid therapy (60mg/day). After imaging diagnosis of abdominal tuberculosis, the patient was started on steroid as well as on ATT. After 5-10 day of starting ATT, the patient went into hypertension (190/100mm of $\mathrm{Hg}$ ). Subsequently steroids were tapered over next 20 days and patient is now doing well only on ATT with normal blood presser and cortisol levels.
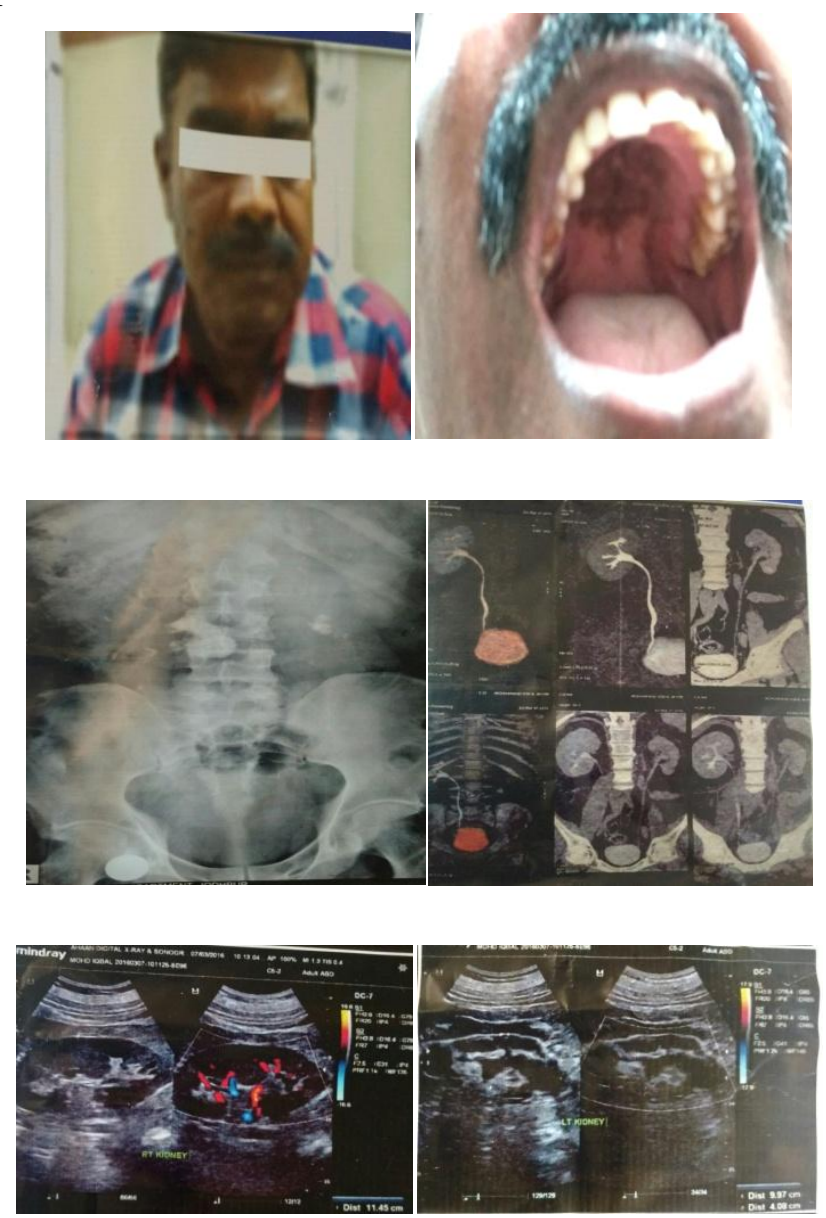

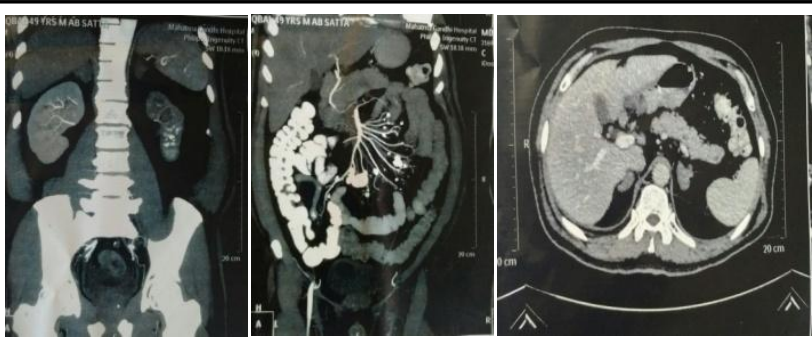

\section{DISCUSSION}

Primary adrenal insufficiency is caused by destruction of the adrenocortical tissue due to autoimmune disease, TB, cryptococcosis, fungal infection and metastatic tumours etc. The resulting adrenal gland destruction may manifest clinically as generalised fatigue, weight loss, fever, dizziness which are nonspecific features of Addison disease. In the extreme situation, an adrenal crisis may occur as a result of infection, surgery or trauma, manifesting as low blood pressure, hyponatremia, hyperkalaemia and/or hypoglycaemia, many of these features present in this case. The symptoms of primary Addison disease manifest when more than $90 \%$ of adrenal gland have been destroyed. For this reason Addison disease due to TB manifest relatively late in life, predominantly in persons aged 40 to 60 years. It has been reported that that major CT finding of adrenal gland $\mathrm{TB}$ are the bilateral enlargement of adrenal glands with calcification. When adrenal TB is active, CECT shows increased enhancement of the peripheral rim of the gland with gland centre showing a lower degree of attenuation. On the other hand, idiopathic Addison disease is not characterised by either adrenal enlargement or calcification on CT. adrenal gland enlargement on CT is frequently recognised within a year of the disease onset. However, the enlarged gland gradually shrinks because of fibrosis and calcification.

\section{CONCLUSION}

Adrenal TB is a rare but important disease entity that must be identified early and treated promptly and aggressively. Adrenal TB patient may present with adrenal insufficiency. advanced imaging like CT abdomen can lead to early diagnosis and 
appropriate treatment of tubercular infection as well as adrenal insufficiency as demonstrated in our patient.

\section{REFERENCES}

1. E. Bhatia, S. K. Jain, R. K. Gupta, and R. Pandey, "Tuberculous Addison's disease: Lack of normalization of adrenocortical function after anti-tuberculous chemotherapy," Clinical Endocrinology, vol. 48, no. 3, pp. 355-359, 1998.

2. E. Charmandari, N. C. Nicolaides, and G. P. Chrousos, Adrenal Insufficiency, Lancet, 2014.

3. F. Kelestimur, "The endocrinology of adrenal tuberculosis: The effects of tuberculosis on the hypothalamo-pituitaryadrenocortical function," Journal of Endocrinological Investigation, vol. 27, no. 4, pp. 380-386, 2004.

4. F. Kelestimur, Y. Unlu, M. Ozesmi, and I. Tolu, "A hormonal and radiological evaluation of adrenal gland in patients with acute or chronic pulmonary tuberculosis," Clinical Endocrinology, vol. 41, no. 1, pp. 53-56, 1994.

5. K. Y. Lam and C. Y. Lo, "A critical examination of adrenal tuberculosis and a 28-year autopsy experience of active tuberculosis," Clinical Endocrinology, vol. 54, no. 5, pp. 633-639, 2001.

6. J. F. McMurry Jr., D. Long, R. McClure, and T. A. Kotchen, "Addison's disease with adrenal enlargement on computed tomographic scanning. Report of two cases of tuberculosis and review of the literature," The American Journal of Medicine, vol. 77, no. 2, pp. 365-368, 1984. 